\title{
In vivo inhibition of neutrophil activity by a FAS (CD95) stimulating module: Arterial in-line application in a porcine cardiac surgery model
}

\author{
Martin Scholz, $\mathrm{PhD}^{\mathrm{a}}$ \\ Andreas Simon ${ }^{\mathrm{a}}$ \\ Markus Berg ${ }^{a}$ \\ Alina Maria Schuller ${ }^{\mathrm{a}}$ \\ Meltem Hacibayramoglua \\ Stefan Margraf, MD \\ Alf Theisen, $M D^{\mathrm{b}}$ \\ Joachim Windolf, MD ${ }^{\mathrm{c}}$ \\ Gerhard Wimmer-Greinecker, MD ${ }^{\mathrm{a}}$ \\ Anton Moritz, MD
}

From the Department of Thoracic and Cardiovascular Surgery ${ }^{\mathrm{a}}$ and the Central Animal Care and Research Facility, ${ }^{\mathrm{b}}$ Johann Wolfgang Goethe University, Frankfurt am Main, Germany, and the Department of Trauma, Hand, and Reconstructive Surgery, ${ }^{\mathrm{c}}$ Hamburg University School of Medicine, Hamburg, Germany.

Received for publication April 24, 2003; revisions received Sept 8, 2003; accepted for publication Sept 10, 2003.

Address for reprints: Martin Scholz, PhD, Department of Thoracic and Cardiovascular Surgery, J.W. Goethe University, Theodor-Stern-Kai 7, D-60590 Frankfurt am Main, Germany (E-mail: m.scholz@ em.uni-frankfurt.de).

J Thorac Cardiovasc Surg 2004;127: 1735-42

$0022-5223 / \$ 30.00$

Copyright () 2004 by The American Association for Thoracic Surgery

doi:10.1016/j.jtcvs.2003.09.027
Objective: Cardiac surgery with cardiopulmonary bypass is associated with aberrant neutrophil activation and potentially severe pathogenic sequelae. This experimental study was done to evaluate a leukocyte inhibition module that rapidly inactivates neutrophils through CD95 stimulation.

Methods: German landrace pigs (4 groups, each $\mathrm{n}=5$ ) underwent cardiac surgery without cardiopulmonary bypass (group I), with cardiopulmonary bypass (group II), with cardiopulmonary bypass plus a leukocyte filter (group III), and with cardiopulmonary bypass plus a leukocyte inhibition module (group IV). The leukocyte filter or leukocyte inhibition module was introduced into the arterial line of the heart-lung machine.

Results: Leukocyte counts were decreased by up to $43 \%$ in group IV compared with values in group II $(P=.023)$. In group IV, but not in groups I to III, no delay in spontaneous neutrophil apoptosis was observed after annexin $\mathrm{V}$-propidium iodide staining. Late apoptotic (11.7\%) or necrotic neutrophils (9.3\%) were detected in 2 animals (group IV). Tumor necrosis factor $\alpha$ serum levels increased over time in groups I to III ( $>2$-fold) but remained at baseline levels in group IV $(P<.05)$. Interleukin 8-mediated chemotactic neutrophil transmigration activity increased over time in groups I to III but was totally abrogated in group IV at any time point. The perioperative increase of creatine kinase and creatine kinase MB levels was lower in groups III (1.5-fold and 1.3-fold, respectively) and IV (1.2-fold and 1.5 -fold, respectively) compared with values in group II (both 1.9-fold).

Conclusions: The leukocyte inhibition module downregulated cardiopulmonary bypass-related neutrophil activity and thus might be beneficial in cardiac surgery and other clinical settings with unappreciated neutrophil activation.

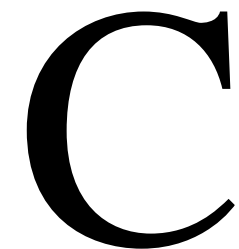

ardiac surgery with cardiopulmonary bypass (CPB) is associated with a broad array of disorders. One major contributing pathogenic mechanism is the aberrant activation of the immune system elicited by the contact of patient blood with artificial surfaces of the extracorporeal circuits. ${ }^{1,2}$ Activated neutrophils are known to elicit endothelial dysfunction through secretion of proteolytic enzymes, such as elastase or oxygen radicals, followed by edema and tissue destruction. ${ }^{3,4}$ 
In the past, several efforts have been undertaken to limit neutrophil activity in cardiac surgery through pharmacologic, ${ }^{5}$ immunologic, ${ }^{6}$ and mechanical ${ }^{7}$ approaches. However, these attempts resulted in many publications with contradictory results and limited success. Leukocyte filtration has been adapted from the transfusion services to reduce the absolute numbers of activated neutrophils. ${ }^{8}$ However, this strategy does not necessarily affect the pathogenic activity of the neutrophils. Recently, our own clinical studies with leukocyte filters (LFs) focused on the efficacy to impair the functional neutrophil activity, such as phagocytic activity, oxidative burst, and elastase levels in the blood. In these trials with high-risk patients, no significant impairment of functional neutrophil activity was found, and a clinical benefit was missing. ${ }^{9}$ Moreover, levels of polymorphonuclear elastase (PMNE), a marker for activated neutrophils, were significantly increased in the filter group versus $\mathrm{CPB}$ alone, suggesting that mechanical stress and the contact with the artificial surface within the filter might stimulate the trapped neutrophils. It is likely that these activated neutrophils are washed out of the filter and circulate in the blood stream. Subsequently, neutrophils might attach to the endothelial wall and transmigrate into subcellular spaces, in which PMNE might disrupt the extracellular matrix and the endothelial integrity.

Recently, we showed in vitro that neutrophils binding to the membrane-bound cell-surface protein Fas ligand (FasL; CD95L, APO-1L) on retinal epithelial cells detached within minutes and lost the ability to adhere and transmigrate. ${ }^{10}$ Interestingly, this loss of neutrophil function occurred independently of neutrophil apoptosis. FasL belongs to the tumor necrosis factor (TNF) family and associates with its receptor Fas (CD95, APO-1). The Fas/FasL system is known as one of the major apoptosis-inducing pathways and a key regulator in natural innate immunity. ${ }^{11}$ However, we found that Fas-mediated leukocyte inhibition preceded early signs of apoptosis.

From these findings, we speculated that the stimulation of Fas on activated neutrophils might be an effective tool to prevent unwanted leukocyte activity and tissue damage in the clinical setting of cardiac surgery with CPB. Therefore we developed a leukocyte inhibition module (LIM) that can be located within the arterial line of the heart-lung machine. The LIM does not retain leukocytes but modifies their function by means of a transient contact with the immunemodulating biomembrane inside.

Here we report our data on the in vivo evaluation of the LIM in a porcine model for cardiac surgery and CPB.

\section{Materials and Methods}

\section{Porcine Model and CPB}

The investigation conforms with the "Guide for the Care and Use of Laboratory Animals" published by the US National Institutes of Health (publication no. 85-23, revised 1996). Pigs (German land- race, $50 \mathrm{~kg}$ ) were allocated to 4 groups (each $\mathrm{n}=5$ ). All pigs were sham operated (median sternotomy) without (group I) or with (groups II-IV) CPB. Anesthesia was maintained consistently with sufentanyl, pancuronium, or propofol. Ventilation was performed with an $\mathrm{O}_{2} / \mathrm{N}_{2} \mathrm{O}$ mixture establishing a fraction of inspired oxygen of 0.5 and a $\mathrm{PCO}_{2}$ of 35 to $40 \mathrm{~mm} \mathrm{Hg}$. After anticoagulation by means of systemic administration of $300 \mathrm{IU} / \mathrm{kg}$ heparin (Liquemin; Roche, Grenzach-Wyhlen, Germany), CPB (crossclamp time, 60 minutes; reperfusion, 30 minutes) was instituted with a Quadrox capillary membrane oxygenator and tubing set, including an arterial filter (Pall, $40 \mu \mathrm{m}$, Dreieich, Germany; group II), an LF (Pall, LG6, group III), or the LIM (LeukoCare, Munich, Germany; group IV). The LIM consists of a thermoplastic module with a volume of $200 \mathrm{~mL}$ housing a Fas (CD95/APO-1)-inducing polyester-based fiber with specific 3-dimensional characteristics that allow blood flow of between 1 and $6 \mathrm{~L} / \mathrm{min}$ and has rheologic features analogous to standard LFs. The 3-dimensional polyester network carries an immobilized immunoglobulin $\mathrm{M}$ anti-Fas antibody (clone CH11; Coulter-Immunotech, Hamburg, Germany) and has a surface of greater than $1 \mathrm{~m}^{2}$. The circuit was primed with $1500 \mathrm{~mL}$ of Ringer's lactate, $500 \mathrm{~mL}$ of $6 \%$ hydroxyethyl starch, $100 \mathrm{~mL}$ of $20 \%$ mannitol, and $150 \mathrm{U} / \mathrm{kg}$ heparin by using a prebypass filter (Pall, $0.2 \mu \mathrm{m}$ ). Additional heparin was administered when the activated clotting time decreased to less than 400 seconds. A flow of $2.4 \mathrm{~L} \cdot \mathrm{min}^{-1} \cdot \mathrm{m}^{-2}$ body surface was applied. The left ventricle was vented through the cardioplegic needle in the ascending aorta. Heparin was fully antagonized with protamine sulfate at the end of CPB. Antegrade cold blood cardioplegia was used (arresting dose, $1000 \mathrm{~mL})$ and reinfused $(400 \mathrm{~mL})$ every 20 minutes.

The operation was terminated by means of controlled potassium infusion.

\section{Blood Sampling}

Blood samples were obtained immediately before the onset of CPB (time point A), 5 minutes before the opening of the aortic crossclamp (time point B), 15 minutes after the opening of the aortic crossclamp (time point $\mathrm{C}$ ), and 10 minutes after weaning from CPB (time point D).

\section{Isolation of Porcine Polymorphonuclear Leukocytes}

The following protocol was used to study whether the LIM might inhibit the assumed pathogenic mechanisms of neutrophils. A volume of $25 \mathrm{~mL}$ of platelet-poor plasma was obtained by centrifuging $50 \mathrm{~mL}$ of heparinized blood at $2500 \mathrm{~g}$ for 10 minutes twice. The plasma was diluted in Hanks buffer (Gibco, Karlsruhe, Germany) solution to reach a concentration of $10 \%$ and used as diluent or washing solution for further procedures.

Twenty milliliters of heparinized whole blood was diluted with $40 \mathrm{~mL}$ of plasma/Hank's buffer solution, carefully layered on top of a 59\% isotonic Percoll (Biochrom, Berlin, Germany) column, and centrifuged at $400 \mathrm{~g}$ for 20 minutes to isolate polymorphonuclear leukocytes (PMNs). The pellet containing red blood cells and PMNs was resuspended $(1: 2 \mathrm{vol} / \mathrm{vol})$ in $2.5 \%$ gelatin containing Hanks buffer (without plasma) and incubated at $37^{\circ} \mathrm{C}$ for 50 minutes. The supernatant was collected and centrifuged at $300 \mathrm{~g}$ for 10 minutes. The remaining red cell pellet was then removed by means of hypotonic hemolysis with $2 \mathrm{~mL}$ of aqua dest (Braun, Melsungen, Germany). PMNs were harvested and washed twice 
with Hank's buffer (without plasma) by centrifuging at $300 g$ for 5 minutes. PMN viability was always greater than $95 \%$, as determined by using Trypan blue exclusion.

\section{Leukocyte Counts}

Blood gas and leukocyte counts were routinely determined with a blood gas analyzer, Cell-Dyn 3500R (Abbott, Wiesbaden Germany).

\section{Enzyme-Linked Immunosorbent Assay}

Serum samples were obtained from porcine blood at time points A through D and stored at $-20^{\circ} \mathrm{C}$. Commercial enzyme-linked immunosorbent assays (ELISAs) were used to determine serum levels of TNF- $\alpha$ (Becton Dickinson, Heidelberg, Germany), creatine kinase (CK), and CK-MB (Roche).

\section{Chemotaxis}

Neutrophils were seeded at a concentration of $1 \times 10^{6} / \mathrm{mL}$ onto 6.5-mm-diameter transwell filters (Corning and Costar, Mainz, Germany) with a pore size of $3 \mu \mathrm{m}$. Cell culture medium in the lower chamber was supplemented with $500 \mathrm{ng} / \mathrm{mL}$ interleukin (IL) 8. After 60 minutes, transwell filters were removed, and neutrophils in the lower chamber were counted microscopically. The amount of transmigrated neutrophils is a measure for the functional activity.

\section{Apoptosis}

Neutrophil apoptosis was determined by using the Apoptosis Detection Kit (R\&D, Wiesbaden, Germany). The principle of this kit is the ability of living cells to expel propidium iodide (PI) and the inability to bind annexin V. Neutrophils were isolated from heparinized blood as described above and incubated $\left(0.5 \times 10^{6} / \mathrm{mL}\right)$ with $0.25 \mu \mathrm{g} / \mathrm{mL}$ fluorescein isothiocyanate (FITC)-conjugated annexin $\mathrm{V}$ and $10 \mu \mathrm{L}$ of PI and analyzed by means of flow cytometry (FACscan, Becton Dickinson). Three subpopulations were identified: viable cells $\left(\mathrm{FITC}^{-} / \mathrm{PI}^{-}\right)$, apoptotic cells $\left(\mathrm{FITC}^{+} /\right.$ $\left.\mathrm{PI}^{-}\right)$, and necrotic cells $\left(\mathrm{FITC}^{+} / \mathrm{PI}^{+}\right)$.

\section{Statistical Analysis}

Data are shown as mean values \pm SD. Repeated-measures analysis of variance (MANOVA) and the Fisher exact test were used for determination of significant differences between groups.

\section{Results}

Microscopic evaluation of the LIM polyester fibers after the end of the operation failed to detect white blood cells (WBCs). In contrast, the LF retained neutrophils that tightly adhered to the polyester fibers (Figure 1).

Leukocyte counts were obtained at defined time points during the operation (Figure 2). Time kinetics showed that during surgical intervention without $\mathrm{CPB}$ (group I), total WBC counts (Figure 2, $A$ ) slightly increased by $18 \%$ at time point $\mathrm{B}, 12 \%$ at time point $\mathrm{C}$, and $5 \%$ at time point $\mathrm{D}$ versus time point $\mathrm{A}\left(13.5 \times 10^{3} / \mu \mathrm{L}\right.$; range, $\left.10.3-16.1 \times 10^{3} / \mu \mathrm{L}\right)$. In animals operated on with CPB (group II), the total WBC counts further increased over time, with a maximum at time

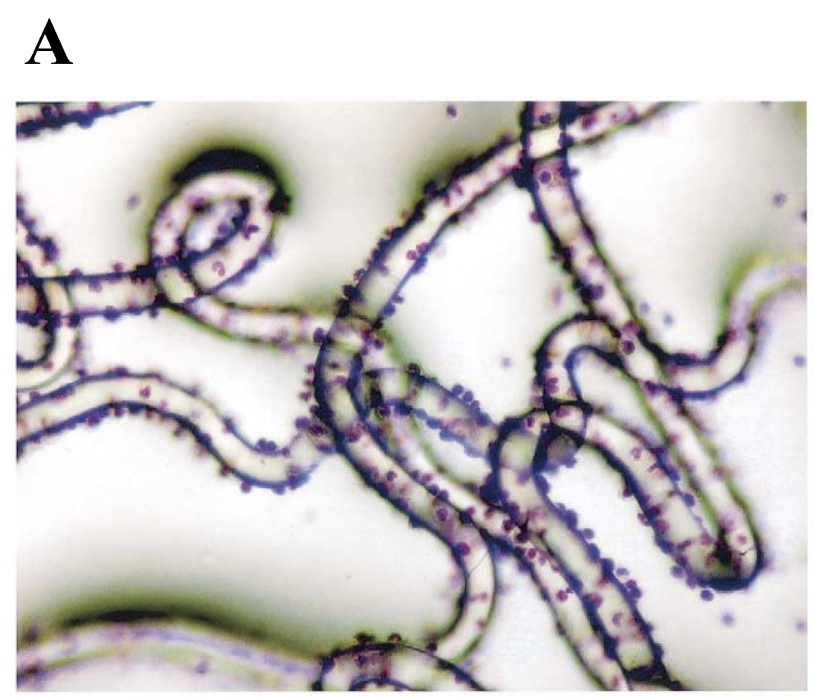

B

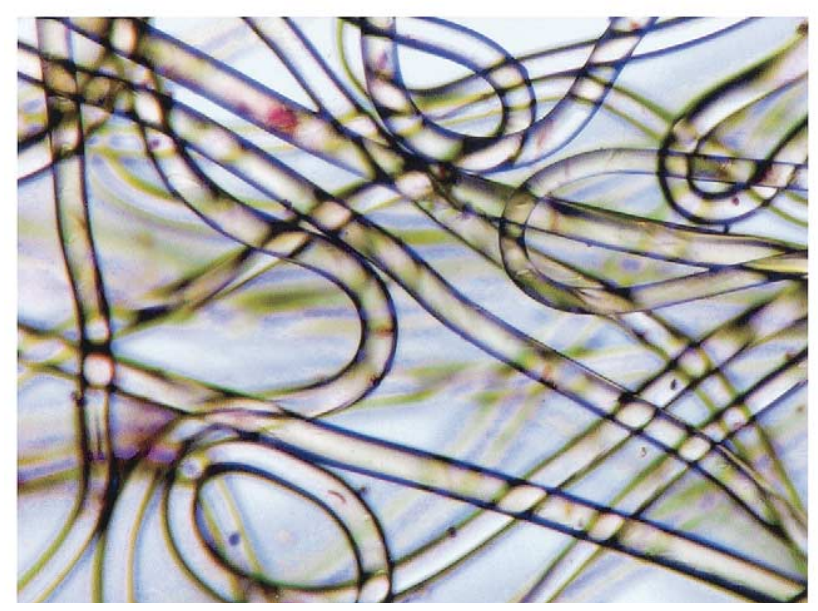

Figure 1. Microscopic evaluation of polyester fibers derived from a standard LF (A) and from the LIM (B) after termination of CPB. Cells were characterized by means of May-Gruenwald/Giemsa staining.

point D (time point B, $28 \%$; time point C, $39 \%$; time point $\mathrm{D}, 76 \%$ vs time point $\mathrm{A}\left(9.4 \times 10^{3} / \mu \mathrm{L}\right.$; range, $4.8-13.2 \times$ $10^{3} / \mu \mathrm{L}$ ). The use of the standard LF LG6 (group III) failed to limit the $\mathrm{CPB}$-associated increase in WBC counts (time point $\mathrm{B},-10 \%$; time point $\mathrm{C}, 21 \%$; time point $\mathrm{D}, 79 \%$ vs time point $\mathrm{A}, 6.5 \times 10^{3} / \mu \mathrm{L}$; range, $\left.4.3-8.6 \times 10^{3} / \mu \mathrm{L}\right)$. Thus no significant differences in WBC counts could be detected between groups II and III. In contrast, when animals were operated on with CPB and LIM (group IV), WBC counts were found to be decreased versus time point $\mathrm{A}(13.1 \times$ $10^{3} / \mu \mathrm{L}$; range, $\left.6.9-17.2 \times 10^{3} / \mu \mathrm{L}\right)$ during the entire observation period (time point $\mathrm{B},-43 \%$; time point $\mathrm{C},-24 \%$; time point $\mathrm{D},-29 \%)$. The difference in time kinetics be- 

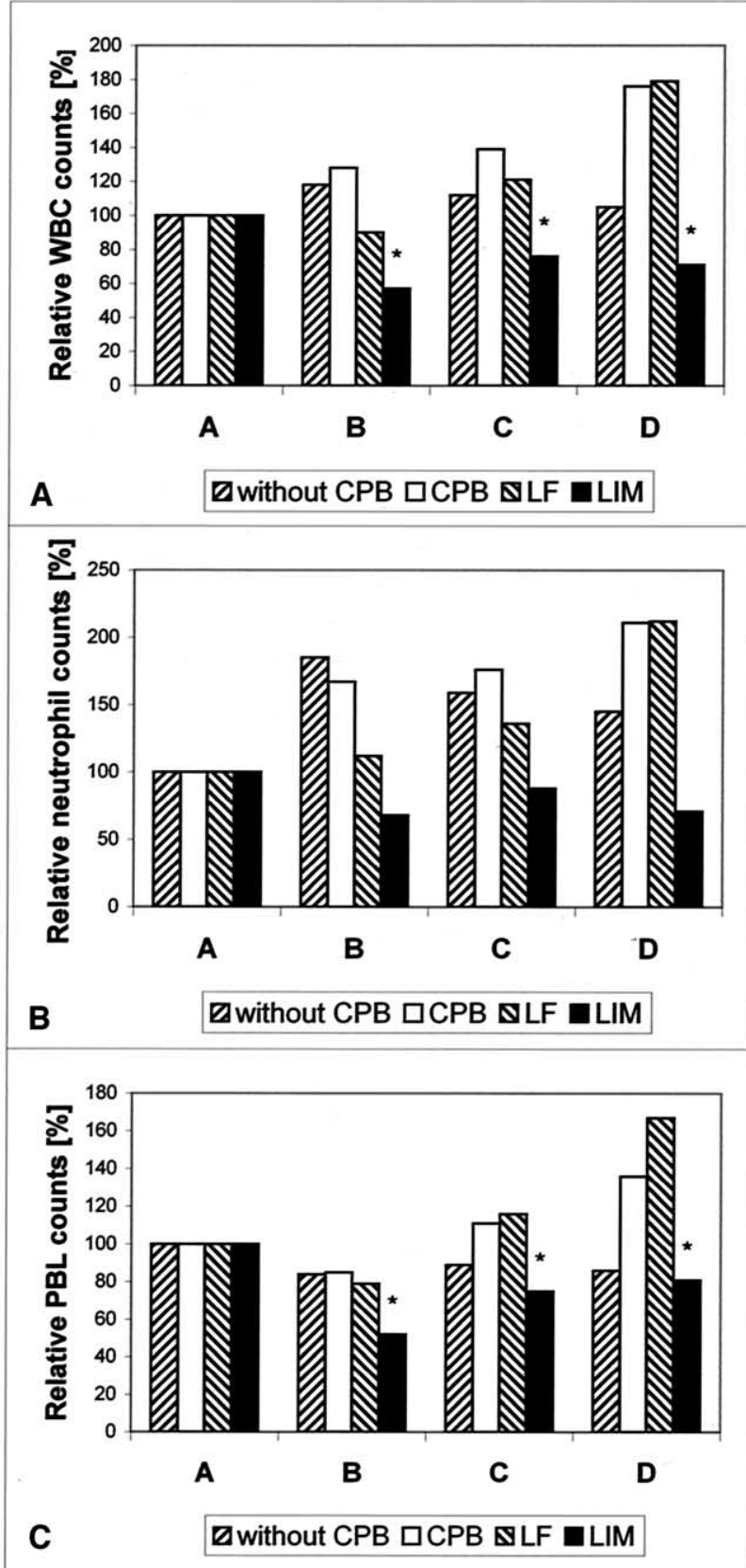

tween groups II and IV was statistically significant $(P=$ .024, MANOVA). However, significant differences were not found between groups for each single time interval.

Overall, similar effects were found for neutrophil counts (Figure 2, B). The neutrophil mean values and ranges at time point A were $4.6 \times 10^{3} / \mu \mathrm{L}\left(3.5-5.3 \times 10^{3} / \mu \mathrm{L}\right)$ for group I, $4.6 \times 10^{3} / \mu \mathrm{L}\left(1.4-7.4 \times 10^{3} / \mu \mathrm{L}\right)$ for group II, 2.4 $\times 10^{3} / \mu \mathrm{L}\left(1.4-4.3 \times 10^{3} / \mu \mathrm{L}\right)$ for group III, and $6.6 \times$ $10^{3} / \mu \mathrm{L}\left(1.8-15.0 \times 10^{3} / \mu \mathrm{L}\right)$ for group IV. In group I neutrophil counts were found to be increased at time point
Figure 2. Time kinetics (time points A, B, C, and D) of leukocyte counts: A, WBCs; B, neutrophils; C, peripheral blood lymphocytes (PBL). Data of different groups (control group without CPB, CPB group, LF group, and LIM group) are shown. Preoperative values (time point $A$ ) were defined as $\mathbf{1 0 0} \%$. Relative data are shown as the mean from 5 independent experiments. The WBC mean \pm SD values at time point $A$ were $13.5 \pm 2.1 \times 10^{3} / \mu \mathrm{L}$ for group $\mathrm{I}, 9.4$ $\pm 5.0 \times 10^{3} / \mu \mathrm{L}$ for group II, $6.5 \pm 1.9 \times 10^{3} / \mu \mathrm{L}$ for group III, and $13.1 \pm 8.5 \times 10^{3} / \mu \mathrm{L}$ for group IV. The neutrophil mean values \pm SD at time point A were $4.6 \pm 0.7 \times 10^{3} / \mu \mathrm{L}$ for group I, $4.6 \pm 2.9$ $\times 10^{3} / \mu \mathrm{L}$ for group II, $2.4 \pm 1.1 \times 10^{3} / \mu \mathrm{L}$ for group III, and $6.6 \pm$ $5.5 \times 10^{3} / \mu \mathrm{L}$ for group IV. The PBL mean values \pm SD at time point A were $8.6 \pm 1.4 \times 10^{3} / \mu \mathrm{L}$ for group $\mathrm{I}, 4.6 \pm 2.3 \times 10^{3} / \mu \mathrm{L}$ for group II, $3.8 \pm 0.9 \times 10^{3} / \mu \mathrm{L}$ for group III, and $7.7 \pm 2.4 \times$ $10^{3} / \mu \mathrm{L}$ for group IV. The difference in time kinetics of WBC counts between groups II and IV but not between the other groups was statistically significant $\left({ }^{*} P=.024\right.$, MANOVA). No significant differences between groups were found for neutrophils (MANOVA). As for PBL counts, only the comparison between groups III and IV showed statistical difference $\left(^{*} P=.016\right.$, MANOVA). However, significant differences were not found between groups for each single time interval (Fisher exact test).

$\mathrm{B}$ and subsequently decreased (time point $\mathrm{B}, 85 \%$; time point $\mathrm{C}, 59 \%$; time point $\mathrm{D}, 45 \%$ vs time point $\mathrm{A}$ ). In group II counts increased over time to reach maximum values at time point $\mathrm{D}$ (time point $\mathrm{B}, 67 \%$; time point $\mathrm{C}, 76 \%$; time point $\mathrm{D}, 112 \%$ vs time point $\mathrm{A}$ ). The LF (group III) limited the increase in neutrophil counts at time points B $(12 \%)$ and C $(36 \%)$ but not at time point D $(112 \%)$ versus time point A. When animals were operated on with CPB and LIM (group IV), neutrophil counts were $-32 \%$ (time point B), $-12 \%$ (time point $\mathrm{C}$ ), and $-29 \%$ (time point $\mathrm{D}$ ) versus time point A. However, differences between groups were not statistically significant either in time kinetics or at each time interval.

The peripheral blood lymphocyte mean values and ranges at time point $\mathrm{A}$ were $8.6 \times 10^{3} / \mu \mathrm{L}(6.4-10.1 \times$ $\left.10^{3} / \mu \mathrm{L}\right)$ for group I, $4.6 \times 10^{3} / \mu \mathrm{L}\left(1.3-6.8 \times 10^{3} / \mu \mathrm{L}\right)$ for group II, $3.8 \times 10^{3} / \mu \mathrm{L}\left(2.8-5.3 \times 10^{3} / \mu \mathrm{L}\right)$ for group III, and $7.7 \times 10^{3} / \mu \mathrm{L}\left(5.0-10.1 \times 10^{3} / \mu \mathrm{L}\right)$ for group IV. As for lymphocytes (Fig 2,C), decreased counts were found in group I (time point $\mathrm{B},-16 \%$; time point $\mathrm{C},-11 \%$; and time point $\mathrm{D},-14 \%$ ) versus time point $\mathrm{A}$. In the CPB group increased counts were detected at time points $\mathrm{C}$ and $\mathrm{D}$ (time point $\mathrm{B},-15 \%$; time point $\mathrm{C}, 11 \%$; and time point $\mathrm{D}, 36 \%$ vs time point A). CPB and LG6 (group III) failed to prevent the increase in lymphocyte counts (time point $\mathrm{B},-21 \%$; time point $\mathrm{C}, 16 \%$; and time point $\mathrm{D}, 67 \%$ vs time point $\mathrm{A}$ ). When animals were operated on with CPB and LIM (group IV), lymphocyte counts were reduced (time point $\mathrm{B},-48 \%$; time point $\mathrm{C},-25 \%$; and time point $\mathrm{D},-19 \%$ vs time point A). The differences in lymphocyte counts over time (time kinetics) between groups III and IV (LG6 and LIM) but not between the other groups were statistically significant $(P=$ 
TABLE 1. Percentage of neutrophils undergoing early apoptosis

\begin{tabular}{lllll}
\hline & \multicolumn{4}{c}{ Time point } \\
\cline { 2 - 5 } & A & B & C & D \\
\hline Group I & 49.4 & 15.6 & 29.7 & 19.6 \\
& 64.7 & 32.8 & 34.7 & 25.3 \\
& 55.4 & 25.9 & 49.6 & 56.2 \\
Group II & 79.8 & 37.7 & 48.2 & 33.7 \\
& 72.1 & 35.6 & 27.9 & 37.1 \\
& 68.5 & 18.5 & 17.9 & 58.9 \\
& 59.8 & 49.6 & 42.2 & 44.8 \\
Group III & 36.9 & 16.9 & 24.9 & 42.3 \\
& 86.3 & 33.3 & 39 & 38.5 \\
& 54.6 & 12.1 & 32.1 & 39.9 \\
& 91.2 & 21.9 & 52.2 & 67.1 \\
Group IV & 66.8 & 28.5 & 44.8 & 45.7 \\
& 50.1 & 14.9 & 32.4 & 62.7 \\
& 72.8 & 22.6 & 29.3 & 35.3 \\
& 69.3 & 34 & 52.7 & 48.1 \\
& 77.3 & 89.5 & 75.2 & 76.6 \\
& 65.9 & 64.7 & 58.3 & 54.8 \\
& 52.6 & 69 & 79.3 & 68.4 \\
& 68.1 & 62.2 & 52.4 & 57.2 \\
& 82 & 79.4 & 57.4 & 54.6 \\
\hline
\end{tabular}

Blood samples were obtained immediately before onset of CPB (time point A), 5 minutes before opening of the aortic crossclamp (time point $B$ ), 15 minutes after opening of the aortic crossclamp (time point $\mathrm{C}$ ), and 10 minutes after weaning from CPB (time point D). Statistical differences between groups were not found, except between groups II and IV at time points $B$ and $C(P<.05$, Fisher exact test $)$.

.016, MANOVA). No statistically significant differences were found when single time intervals were compared between groups (Fisher exact test).

Because activated neutrophils might elicit severe pathogenic sequelae during cardiac surgery with $\mathrm{CPB}$, we further focused on the functional effects of the LIM on neutrophil activity. First, we studied, by means of flow cytometry, whether cardiac surgery and CPB with or without the LIM might impair the ability of neutrophils to undergo spontaneous apoptosis. In all blood samples obtained from groups I to IV (Table 1), spontaneous neutrophil apoptosis (annexin $\mathrm{V}^{+} / \mathrm{PI}^{-}$) was detected at time point $\mathrm{A}$ (range, $49.4 \%$ $91.2 \%$ ). In groups I to III the percentage of annexin $\mathrm{V}^{+} / \mathrm{PI}^{-}$ cells decreased by up to $80 \%$ over time (time points B-D). In contrast, in group IV the percentage of annexin $\mathrm{V}^{+} / \mathrm{PI}^{-}$ neutrophils increased $(n=2)$, for example, from $77.3 \%$ at time point $\mathrm{A}$ to $89.5 \%$ (time point $\mathrm{B}$ ) and remained around baseline level (time point $\mathrm{C}, 75.2 \%$; time point $\mathrm{D}, 76.6 \%$ ). Moreover, in 2 animals of group IV but in none of groups I to III, late apoptosis (annexin $\mathrm{V}^{+} / \mathrm{PI}^{+} ; 11.7 \%$ and $3.72 \%$ ) at time point $\mathrm{C}$ and necrosis (annexin $\mathrm{V}^{-} / \mathrm{PI}^{+} ; 9.0 \%$ and 9.3\% at time point D) were found. Statistical differences between groups were not found, except between groups II and IV at time points $\mathrm{B}$ and $\mathrm{C}(P<.05$, Fisher exact test).

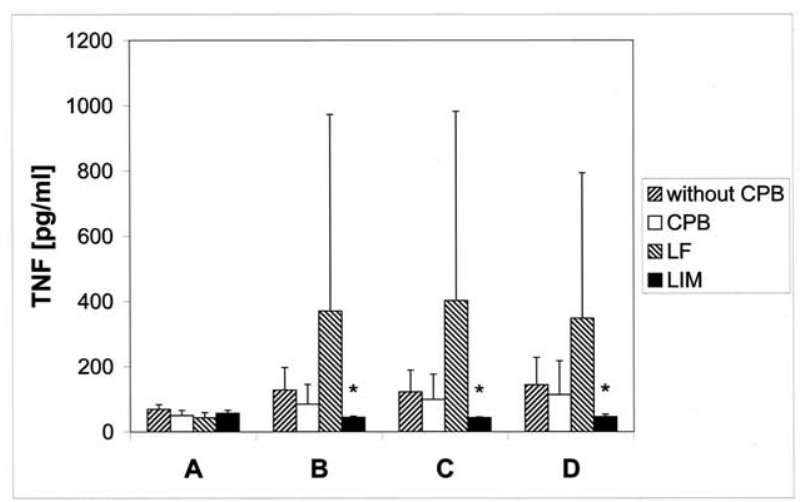

Figure 3. Serum levels of TNF- $\alpha$ in each group depicted as time kinetics (time points A-D). Data are shown as the mean $(n=5) \pm$ SD. ${ }^{*} P<.05$ for group IV (LIM) versus groups II and III (CPB and LF).

To find out whether the LIM might prevent the secretion of proinflammatory cytokines, TNF- $\alpha$ levels were serially determined in porcine blood samples by means of ELISA. Whereas TNF- $\alpha$ levels increased over time in groups I to III ( $>200 \%$ at time point D), no enhanced levels were found in group IV (time point A, $57.4 \pm 9.0 \mathrm{pg} / \mathrm{mL}$; time point $\mathrm{B}$, $43.6 \pm 3.5 \mathrm{pg} / \mathrm{mL}$; time point $\mathrm{C}, 43.2 \pm 3.2 \mathrm{pg} / \mathrm{mL}$; time point $\mathrm{D}, 46.4 \pm 7.8 \mathrm{pg} / \mathrm{mL}$ ). In group III (LF) extremely high TNF- $\alpha$ values in 2 of 5 animals were detected (mean values $>800 \%$ ). Differences between groups II and IV and between groups III and IV at time points $\mathrm{B}, \mathrm{C}$, and $\mathrm{D}$, but not at time point $\mathrm{A}$, were statistically significant $(P<.05)$. Mean \pm SD values are shown in Figure 3 for all groups.

As a further functional parameter, neutrophil IL-8-directed chemotactic migration was evaluated. Over time (time points A-D), the numbers of transmigrating neutrophils increased in groups II $(P=.036$, MANOVA $)$ and III $(P=.044$, MANOVA $)$ compared with in group I. Maximum values were found in group II at time point D (451 \pm 250; range, 165-850). In group IV neutrophil transmigration was totally abrogated at time points $\mathrm{B}, \mathrm{C}$, and $\mathrm{D}(P<.05$ vs group II but not group III, Fisher exact test). Figure 4 shows absolute and mean data ( $\mathrm{n}=5$, groups I-IV) obtained with neutrophils isolated at time points A to D.

As tissue damage parameters, systemic CK and myocardium-specific isoenzyme CK-MB levels were determined by means of ELISA. As shown in Figure 5, CK and CK-MB levels increased 1.3-fold and 1.4-fold in group I, respectively, whereas both markers increased 1.9-fold over time (time point $\mathrm{D}$ ) when animals were operated on with $\mathrm{CPB}$ (group II). In groups III and IV the CPB-associated increase of CK and CK-MB was limited (group III, 1.5-fold and 1.3-fold; group IV, 1.2-fold and 1.5-fold). Differences between groups II and IV at time points $\mathrm{B}$ and $\mathrm{C}$ but not $\mathrm{A}$ and D were statistically significant $(P<.05$; Fisher exact test). 


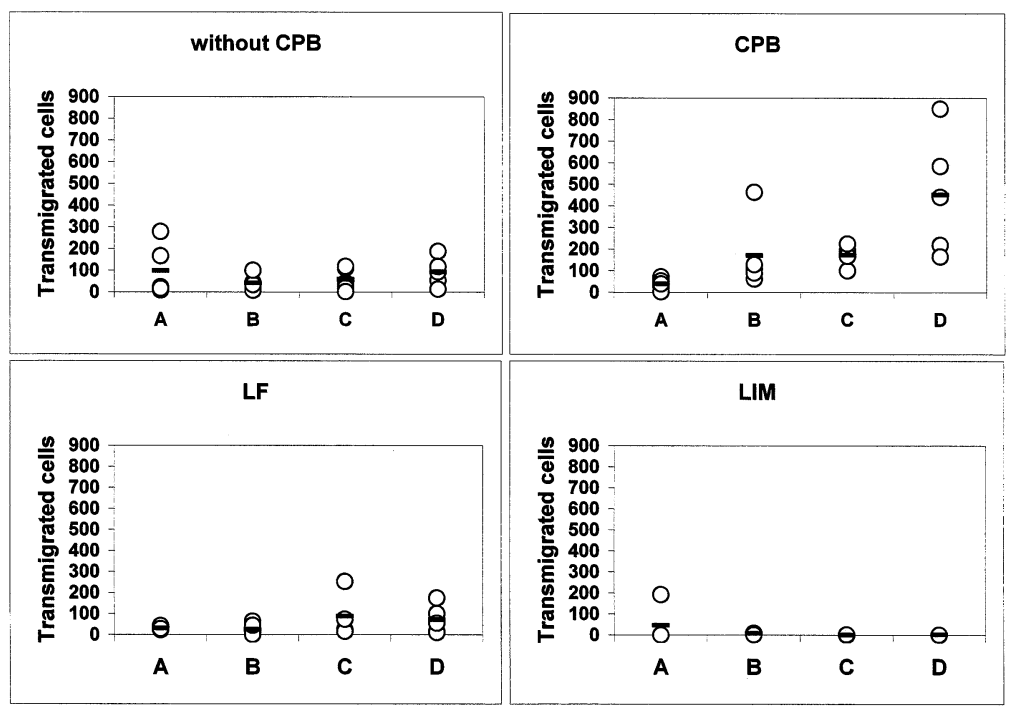

Figure 4. Time kinetics of neutrophil IL-8-directed chemotaxis, as determined in transwell chambers. Neutrophils were isolated from blood obtained at time points $A$ to $D$ from different groups (without $C P B$, with CPB alone, CPB plus LF, and CPB plus LIM). Data are depicted as the absolute numbers of transmigrated cells that were counted in the lower chamber. The results of each measurement are shown as open circles, and the mean values are shown as bars ( $\mathrm{n}=5$ in each group). Differences between groups II and IV but not between groups III and IV were statistically significant at time points $B, C$, and $D(P<.05$, Fisher exact test).
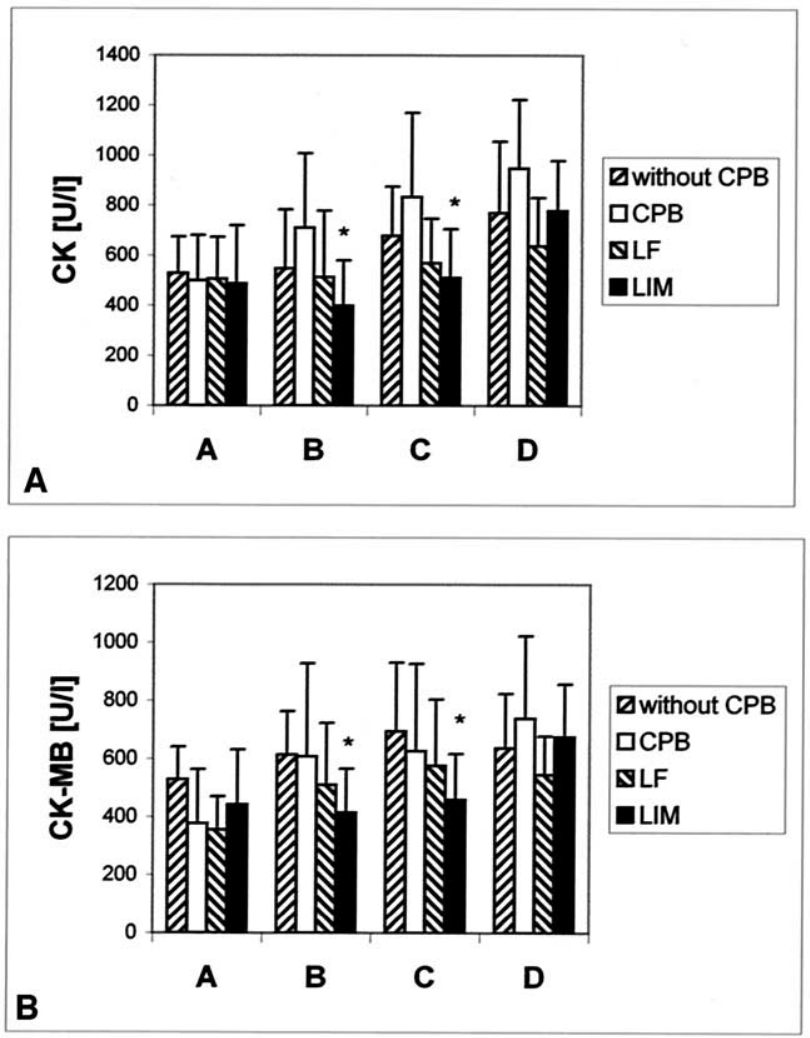

Figure 5. Serum levels of CK (A) and CK-MB (B) are depicted as time kinetics for each group. Data are shown as the means $(\mathrm{n}=$ 5) \pm SD. ${ }^{*} P<.05$ for the LIM group versus the CPB group (Fisher exact test).

\section{Discussion}

Highly activated neutrophils might cause severe pathogenic sequelae related to cardiac surgery with CPB. Therefore the inhibition of neutrophil activity is an important goal to achieve. Here we have shown that the LIM, but not leukocyte filtration, overall prevented neutrophil activity during cardiac surgery in a porcine model. The LIM was found to manipulate leukocyte functions within the blood stream.

The theoretic approach for the development of the LIM was the in vitro finding that neutrophils that attach to FasL (CD95L or APO-1L) expressing epithelial cells through Fas (CD95) lose their cell-to-cell contacts within minutes and fail to transmigrate the epithelial barrier. ${ }^{10}$ This unexpected finding was found to be independent from Fas/FasL-mediated apoptosis, which is known to play an important physiologic role in the regulation of innate immune responses. ${ }^{11}$ FasL belongs to the TNF family and is the ligand of Fas that is expressed on activated leukocytes and on other tissue cells, especially in immune-privileged sites. ${ }^{12}$ The interaction of FasL with Fas triggers apoptosis signaling and cell death. Deregulated Fas/FasL-dependent mechanisms are involved in many autoimmune and inflammatory diseases, such as Hashimoto thyroiditis, ${ }^{13}$ hepatitis, ${ }^{14}$ or myasthenia gravis. ${ }^{15}$ Therapeutically, soluble Fas has been used to prevent hepatitis B in mice $^{14}$ or for the treatment of systemic lupus erythematosus. ${ }^{16}$ Moreover, monoclonal antibodies against CD95 (anti-Fas) have been used to induce apoptosis in tumor cells ${ }^{17}$ and to kill HIV-infected cells. ${ }^{18}$ 
Unfortunately, local induction of apoptosis or administration of anti-Fas antibodies, for example in the lung, have been reported to induce severe inflammatory and fibrotic disorders. ${ }^{19,20}$ In contrast to these previous approaches, we here report on anti-Fas-mediated effects that are limited to an extracorporeal device. Experiments with radioactivelabeled antibodies showed that binding to the LIM surface is stable, even under adequate flow conditions (not shown). Thus the LIM allows the inactivation of floating neutrophils after a short transient contact with the Fas-stimulating polyester fiber surface without affecting other tissues by means of apoptosis-induced inflammation. Inactivation occurs within minutes, and inactive neutrophils immediately can be recognized and ingested by macrophages. ${ }^{21}$

Experimentally, apoptosis normally is not detectable before 4 hours after Fas/FasL stimulation, ${ }^{10}$ and thus the functional neutrophil inhibition observed in our in vivo study is likely to occur independent of apoptosis. However, we compared ex vivo the percentage of neutrophils that undergo spontaneous apoptosis among groups 4 hours after neutrophil isolation. These experiments revealed that the surgical procedures (groups I-III) decreased the percentage of spontaneous early apoptotic neutrophils compared with neutrophils isolated before the operation. In 2 of 5 animals, the LIM prevented the decrease in apoptotic neutrophils, suggesting that the LIM induces early apoptosis through CD95 stimulation. Impaired apoptosis, delayed apoptosis, or both is known to be associated with proinflammatory diseases, such as Kawasaki disease ${ }^{22}$ and systemic inflammatory response syndrome. ${ }^{23}$ Accordingly, in cardiac surgery delayed apoptosis of neutrophils has been associated with inflammatory disorders ${ }^{24}$ and thus CD95 stimulation by the LIM might be an adequate tool to prevent these disorders. However, preliminary annexin V/PI data obtained with whole blood immediately after collection currently does not provide evidence for LIM-mediated rapid induction of apoptosis (unpublished data). Therefore we suggest that the observed Fas-related inhibition of neutrophil activity precedes apoptosis, a phenomenon that has been described recently. ${ }^{10}$

Further studies now have to evaluate in detail the fate of leukocytes that have passed the LIM in a quantitative manner. In addition, further investigations into the molecular mechanisms are needed. We speculate that Fas signalingrelated phenotypic cellular changes ${ }^{25}$ might lead to a rapid clearance of inactive-preapoptotic neutrophils by phagocytes. ${ }^{21}$ This would explain the reduced leukocyte numbers observed in the LIM group but not in the LF group, although we found trapped neutrophils within the filter mesh of the LF but almost no leukocytes within the LIM. The increase of WBCs and neutrophils over time in groups I and II but also in the LF group might be explained by the intraoperative release of neutrophil precursors from the bone marrow. ${ }^{7}$
The LF probably reduced leukocyte counts only transiently during the early phase of CPB, which is in accordance with findings from clinical studies. ${ }^{26}$ Moreover, the lymphocyte population was found to be significantly reduced in the LIM versus LF group, which also might be due to CD95-mediated inactivation. ${ }^{10}$ It has been reported that surgical trauma and $\mathrm{CPB}$ modulate leukocyte counts, and this function might contribute to CPB-related immunosuppression and increased incidence of postoperative infectious complications. ${ }^{27}$ Our data indicate that the LIM stabilizes the lymphocyte population at least regarding the lymphocyte counts at the end of the operation. Moreover, mitogen-induced lymphocyte proliferation was found at any time point in either group, indicating that the LIM does not impair functional activity of lymphocytes (not shown).

In contrast, the LIM significantly limited the levels of the proinflammatory cytokine TNF- $\alpha$, which is known to contribute to the development of systemic inflammatory response syndrome ${ }^{28}$ and epithelial barrier dysfunction. ${ }^{29}$ The LIM therefore might be an important tool to prevent TNF$\alpha$-related pathogenesis without pharmacologic intervention $^{30}$ in cardiac surgery but also in other clinical settings, such as transplantation, trauma, or sepsis. ${ }^{28}$ Preliminary results in a similar experimental setting showed that in addition to TNF- $\alpha$, CPB-mediated increases in IL- 6 and IL-8 levels are also prevented by the LIM (unpublished data).

In addition, endothelial leakage and extracellular matrix destruction might result from neutrophil adhesion and transendothelial migration. ${ }^{3,4}$ In our transwell chamber assay, we found a significant CPB-mediated increase of IL-8-directed neutrophil transmigration activity over time. Surprisingly, neutrophils in the LIM group lost their ability to transmigrate in this model. This finding, in conjunction with the failure to detect adherent neutrophils within the LIM, confirms recently published in vitro data showing neutrophil inactivation after short contact with FasL-expressing epithelial cells. ${ }^{10}$ The underlying signaling and biochemical pathways downstream from Fas that lead to the rapid functional loss are thus far unknown.

Overall, evidence was found that the LIM protected from CPB-associated pathogenicity because CK and CK-MB values were reduced compared with those with $\mathrm{CPB}$ alone. Although similar findings were observed in the LF group, in a previous clinical trial with high-risk patients and an LF, we found rather increased values for CK and CK-MB in the filter group (unpublished data) in conjunction with significantly increased PMNE values. ${ }^{9}$ Unfortunately, we were not able to measure porcine PMNE because of the lack of commercially available porcine PMNE in ELISA.

The reason for the rather negative effects of the LF observed in this study are unclear. However, we suppose that neutrophils that are trapped within the filter mesh are 
still active and secrete proinflammatory cytokines that in turn stimulate unappreciated immune functions. The finding that the LF, in spite of the failure to reduce overall leukocyte counts, reduced CK and CK-MB levels on the other hand might be due to the LF feature of trapping a significant number of activated neutrophils, thus preventing neutrophil adhesion to the endothelium and subsequent tissue damage. It is surprising that the LIM also limits CK and CK-MB increase without trapping neutrophils, underlining the efficient CD95-mediated neutrophil inactivation within the LIM.

Ongoing studies in the same experimental setup revealed that the LIM elicits positive effects on the postoperative cardiac function (unpublished). However, we were not able to show whether the LIM has beneficial effects on pulmonary, renal, and liver function because in our model CPBmediated decrease in organ function could not be observed. On the cerebral level, the LIM did not prevent CPB-mediated leakage of the porcine blood-brain barrier, as detected with Evan's blue injection and postoperative histologic and photometric evaluation. However, the relevance of this finding is unclear because cerebral edema could not be detected by means of magnetic resonance tomography (data not shown).

In conclusion, in our porcine model the LIM proved to be an effective tool to functionally inhibit leukocyte activation and thus to limit pathogenic sequelae related to cardiac surgery with CPB. The LIM allows the inactivation of neutrophils through Fas stimulation without affecting other organs by apoptosis-mediated inflammation. ${ }^{22,23}$ Further studies ought to be conducted to evaluate postoperative benefits and the efficacy of the LIM in clinical studies.

We appreciate the excellent technical assistance of Mrs Vera Beinrucker and Mrs Alexandra Best. For statistical analyses, we are grateful to Dr Sonia Area de Leao Sitals.

\section{References}

1. Kirklin JK, Westaby S, Blackstone EH, Kirklin JW, Chenoweth DE, Pacifico AD. Complement and the damaging effects of cardiopulmonary bypass. J Thorac Cardiovasc Surg. 1983;86:845-57.

2. Butler J, Rocker GM, Westaby S. Inflammatory response to cardiopulmonary bypass. Ann Thorac Surg. 1993;55:552-9.

3. Carden D, Xiao F, Moak C, Willis BH, Robinson-Jackson S, Alexander S. Neutrophil elastase promotes lung microvascular injury and proteolysis of endothelial cadherins. Am J Physiol. 1998;275:H385-92.

4. Welbourn CR, Goldman G, Paterson IS, Valeri CR, Shepro D, Hechtman HB. Neutrophil elastase and oxygen radicals: synergism in lung injury after hindlimb ischemia. Am J Physiol. 1991;260:H1852-6.

5. El Azab SR, Rosseel PM, de Lange JJ, et al. Dexamethasone decreases the pro- to anti-inflammatory cytokine ratio during cardiac surgery. Br J Anaesth. 2002;88:496-501.

6. Sablotzki A, Muhling J, Dehne MG, Zickmann B, Silber RE, Friedrich I. Treatment of sepsis in cardiac surgery: role of immunoglobulins. Perfusion. 2001;16:113-20.

7. Matheis G, Moritz A, Scholz M, eds. Leukocyte depletion in cardiac surgery and cardiology. Basel: Karger; 2001.
8. de Vries AJ, Gu YJ, Post WJ, et al. Leucocyte depletion during cardiac surgery: a comparison of different filtration strategies. Perfusion. 2003;18:31-8.

9. Scholz M, Simon A, Matheis G, et al. Leukocyte filtration fails to limit functional neutrophil activity during cardiac surgery. Inflamm Res. 2002;51:363-8.

10. Cinatl J Jr, Blaheta R, Bittoova M, et al. Decreased neutrophil adhesion to human cytomegalovirus-infected retinal pigment epithelial cells is mediated by virus-induced up-regulation of Fas ligand independent of neutrophil apoptosis. J Immunol. 2000;165:4405-13.

11. Rowe PM. Glimmers of clinical relevance for Fas. Lancet. 1996;347: 1398.

12. Gregory MS, Repp AC, Holhbaum AM, et al. Membrane Fas ligand activates innate immunity and terminates ocular immune privilege. J Immunol. 2002;169:2727-35.

13. Giordano C, Stassi G, De Maria R, et al. Potential involvement of Fas and its ligand in the pathogenesis of Hashimoto's thyroiditis. Science. 1997;275:960-3

14. Kondo T, Suda T, Fukuyama H, Adachi M, Nagata S. Essential roles of the Fas ligand in the development of hepatitis. Nat Med. 1997;3: 409-13.

15. Moulian N, Bidault J, Truffault F, Yamamoto AM, Levasseur P, Berrih-Aknin S. Thymocyte Fas expression is dysregulated in myasthenia gravis patients with anti-acetylcholine receptor antibody. Blood. 1997;89:3287-95.

16. Cheng J, Zhou T, Liu C, et al. Protection from Fas-mediated apoptosis by a soluble form of the Fas molecule. Science. 1994;263:1759-62.

17. Trauth BC, Klas C, Peters AM, et al. Monoclonal antibody-mediated tumor regression by induction of apoptosis. Science. 1989;245:301-5.

18. Kobayashi N, Hamamoto Y, Yamamoto N, Ishii A, Yonehara M, Yonehara S. Anti-Fas monoclonal antibody is cytocidal to human immunodeficiency virus-infected cells without augmenting viral replication. Proc Natl Acad Sci U S A. 1990;87:9620-4.

19. Cox G, Crossley J, Xing Z. Macrophage engulfment of apoptotic neutrophils contributes to the resolution of acute pulmonary inflammation in vivo. Am J Respir Cell Mol Biol. 1995;12:232-7.

20. Hagimoto N, Kuwano K, Miyazaki H, et al. Induction of apoptosis and pulmonary fibrosis in mice in response to ligation of Fas antigen. Am J Respir Cell Mol Biol. 1997;17:272-8.

21. Vandivier RW, Fadok VA, Hoffmann PR, et al. Elastase-mediated phosphatidylserine receptor cleavage impairs apoptotic cell clearance in cystic fibrosis and bronchiectasis. J Clin Invest. 2002;109:661-70.

22. Tsujimoto H, Takeshita S, Nakatani K, Kawamura Y, Tokutomi T, Sekine I. Delayed apoptosis of circulating neutrophils in Kawasaki disease. Clin Exp Immunol. 2001;126:355-64.

23. Jimenez MF, Watson RW, Parodo J, et al. Dysregulated expression of neutrophil apoptosis in the systemic inflammatory response syndrome. Arch Surg. 1997;132:1263-9.

24. Chello M, Mastroroberto P, Quirino A, et al. Inhibition of neutrophil apoptosis after coronary bypass operation with cardiopulmonary bypass. Ann Thorac Surg. 2002;73:123-9.

25. Barker RN, Erwig LP, Hill KS, Devine A, Pearce WP, Rees AJ. Antigen presentation by macrophages is enhanced by the uptake of necrotic, but not apoptotic, cells. Clin Exp Immunol. 2002;127:220-5.

26. Matheis G, Scholz M, Simon A, Henrich D, Wimmer-Greinecker G, Moritz A. Timing of leukocyte filtration during cardiopulmonary bypass. Perfusion. 2001;16:31-7.

27. Markewitz A, Faist E, Lang S, Endres S, Fuchs D, Reichart B. Successful restoration of cell-mediated immune response after cardiopulmonary bypass by immunomodulation. J Thorac Cardiovasc Surg. 1993;105:15-24.

28. Graninger W, Wenisch C. Pentoxifylline in severe inflammatory response syndrome. J Cardiovasc Pharmacol. 1995;25(suppl 2):S134-8.

29. Suenaert P, Bulteel V, Lemmens L, et al. Anti-tumor necrosis factor treatment restores the gut barrier in Crohn's disease. Am J Gastroenterol. 2002;97:2000-4.

30. Sack M. Tumor necrosis factor-alpha in cardiovascular biology and the potential role for anti-tumor necrosis factor-alpha therapy in heart disease. Pharmacol Ther. 2002;94:123-35. 